\title{
LA ORIENTACIÓN DE LA ESTRUCTURA 33 DE YAXCHILÁN: UNA REEVALUACIÓN"
}

\author{
STANISLAW IWANISZEWSKI \\ División de Posgrado, ENAH-INAH \\ Jesús Galindo Trejo \\ Instituto de Astronomía, UNAM
}

\section{Introducción}

Pájaro Jaguar IV (752-768), el décimo tercer gobernante de Yaxchilán ( $\mathrm{Pa}^{\prime}$ chan, "cielo dividido/roto", Martin, 2004), se entronizó en el año 752 contando con 43 años de edad, al finalizar un período de interregno de casi 10 años que pasaron después de la muerte de su padre, Escudo Jaguar II (Itzamnaaj B'ahlam II, 681-742). Durante la etapa de consolidación de su poder, una de sus preocupaciones fue demostrar la legitimidad de su linaje. Siendo hijo de la Señora Ik' Cráneo de Calakmul (Martin y Grube, 2000: 128), una de las esposas menores de Escudo Jaguar II, Pájaro Jaguar IV mandó hacer una serie de edificios, dinteles y estelas para mostrar que su derecho al trono era resultado de una decisión tomada por su padre. La estela 11 de Yaxchilán representa a Pájaro Jaguar IV sosteniendo el estandarte llamado jasaw chan (Freidel et al., 1993: 271). Los investigadores (ibid.) opinan que la escena representa a Pájaro Jaguar IV, quien, bailando, intercambia los estandartes con su padre, Escudo Jaguar II (Itzamnaaj B'ahlam II, 681-742). El texto colocado a los pies de Escudo Jaguar II apunta: “En (9.15.9.17.16) 12 Kib' 19 Yaxk'in se llevó a cabo la ceremonia del estandarte (por) el ajaw de 5 katunes, Escudo Pájaro II, el ajaw de Yaxchilán” (Mathews, 1997: 177). Posteriormente, gracias a las lecturas de Grube (1992) y Looper (2003) se logró identificar el nombre del baile y traducir esta frase como sigue: “En (9.15.9.17.16) 12 Kib' /Chib’in/ 19 Yaxk'in bailó la danza jasaw chan el ajaw de 5 katunes, Escudo Pájaro II, el ajaw de Yaxchilán ( $\mathrm{Pa}$ ' chan)”. Looper (op. cit.) también sugiere que el nombre del baile, jasaw chan, denota la acción de dividir o aclarar/despejar el cielo durante la llegada de las lluvias, o de marcar la mitad del año (referente a la fecha solsticial que puede servir para señalar la división del año). En efecto, la fecha maya corresponde a la fecha gregoriana del 26 de ju-

\footnotetext{
* El trabajo de campo en Yaxchilán se hizo gracias al apoyo del CONACYT (Beca 25721-S, Proyecto "La arquitectura y la configuración espacio-tiempo en la sociedad maya"). Agradecemos al Consejo de Arqueología por otorgarnos los permisos correspondientes.
} 
nio de $741,{ }^{1}$ o sea, cuatro días después del solsticio de verano. Siguiendo la propuesta original de Proskouriakoff (1963: 164), quien interpretó esta escena en términos de la transferencia del poder entre padre e hijo, Mathews (op. cit.: 177) la denominó como el intercambio de estandartes. Por su lado, Miller (2001: 213) trata de generalizar el ritual de la danza. Observa que estos rituales con frecuencia se realizaban en la región del Usumacinta (incluyendo Piedras Negras, Bonampak y Lacanhá) y posiblemente se relacionaron con el poderío de Yaxchilán. Cabe mencionar que los objetos rituales que los gobernantes sostuvieron durante la danza ritual se conocen con los nombres de jasaw y xukpi. Por otro lado, para Looper (op. cit.) se puede tratar de un acto de la magia utilizada por los gobernantes mayas para propiciar el clima adecuado. En la estela 50 se ve al gobernante conocido como K'inich Tatb’u Cráneo II bailando la danza hab'ab' (de "dividir" o “despojar") en un contexto semejante, y Looper (op. cit.) concluye que se trata de la danza ritual que el gobernante baila cerca del solsticio de verano para señalar la división del año (antes/después del solsticio) y eventualmente la temporada de lluvias y la segunda siembra del maíz. En nuestra opinión, las conclusiones que Looper retoma de Tate no pueden referirse al período de canícula, ya que ésta se inicia a finales de julio, un mes después del solsticio de verano. Concluimos que existe la posibilidad de marcar o señalar el momento de la división del año con respecto al solsticio, pero difícilmente podemos atribuirla al inicio de la canícula.

En este contexto es interesante notar que el significado del nombre de Yaxchilán, Pa' chan, "cielo dividido/roto", enfatiza el concepto de la división o de la ruptura del cielo (Martin, op. cit.: 6). La idea de un cielo dividido o roto aparece ya en las inscripciones tempranas, años antes de la actividad arquitectónica de Pájaro Jaguar IV. No es posible decidir si las colinas que bordean Yaxchilán pueden considerarse como el elemento paisajístico que originó las metáforas para nombrar esta localidad, tal como lo propone Alfonso Lacadena (citado por Martin, ibid.); pero la danza ritual referente al acto de dividir/aclarar/despejar el cielo eventualmente podría derivar su legitimidad del nombre de Yaxchilán. Sea como fuere, los bailes rituales que legitiman la sucesión de los gobernantes también constituyen marcos temporales para dividir el flujo de tiempo en los segmentos menores apropiados por los gobernantes en turno.

Mathews (citado por Justeson, 1989: 114) y Tate (1986, 1989, 1997: 94-95) notaron una serie de inscripciones que hacían referencias a los rituales celebrados durante el mes de Yaxk'in. Al analizar los rituales descritos en las inscripciones en Yaxchilán (estelas 11 y 16, dinteles 9, 33, 50), Carolyn Tate (1986, 1997: 94-95) descubrió que sus fechas, convertidas al calendario juliano, caían cerca del momento del solsticio de verano (véase tabla 1). Esta investigadora (1986: 100-102; 1997: 96) recalcó que los rituales de intercambio de estandar-

\footnotetext{
${ }^{1}$ En este artículo utilizamos la constante de correlación 584, 285.
} 
tes denotaban el paso del poder entre uno y otro gobernante y se hacían cerca de los momentos del solsticio de verano. Al reconocer el valor simbólico del momento solsticial en los rituales de la transferencia del poder, Tate también observó que especialmente durante el reinado de Escudo Jaguar II y Pájaro Jaguar IV los edificios se construyeron orientados hacia los puntos de la salida del sol durante dicho solsticio. Utilizando las mediciones hechas con una brújula, la investigadora estableció que las estructuras 20,21, 33, 40 y 41, atribuidas al reinado de Pájaro Jaguar IV, se orientaban entre $\operatorname{los} 51^{\circ}$ y $54^{\circ}$, y notó que durante los días cercanos al solsticio de verano los rayos solares iluminaban durante un corto momento los interiores de dichos edificios (Tate, 1997: 113). En particular, Tate describió la manera como los rayos solares un momento después de la salida del astro diurno proyectaban luz al interior de la Estructura 33, iluminando la estatua de Pájaro Jaguar IV.

Tabla 1. Los datos Cronológicos relacionados CON LoS Rituales DE INTERCAMBIO DE ESTANDARTE

\begin{tabular}{|l|l|l|l|l|}
\hline monumento & fecha & rueda calendárica & $\begin{array}{c}\text { fecha } \\
\text { gregoriana } \\
(584,285)\end{array}$ & \multicolumn{1}{|c|}{ evento } \\
\hline Estela 11 & 9.15 .9 .17 .16 & 12 Kib 19 Yaxk'in & 26.06 .741 & $\begin{array}{l}\text { E-J II (Itzamnaaj } \\
\text { ''ahlam II) intercambia } \\
\text { el estandarte jasaw } \\
\text { chan con P-J IV }\end{array}$ \\
\hline Estela 16 & $9.15 .4 .16 .11^{1}$ & 7 Chuwen 19 Yaxk'in & 27.06 .736 & $\begin{array}{l}\text { E-J II con el estandarte } \\
\text { jasaw chan }\end{array}$ \\
\hline Dintel 33 & 9.15 .16 .1 .6 & 5 Kimi 19 Yaxk'in & 25.06 .747 & $\begin{array}{l}\text { P-J IV sosteniendo el } \\
\text { estandarte jasaw chan }\end{array}$ \\
\hline Dintel 9 & 9.16 .17 .6 .12 & 1 Eb fin de Yak'in & 20.06 .768 & $\begin{array}{l}\text { P-J IV intercambia el } \\
\text { estandarte jasaw chan } \\
\text { con señor Gran } \\
\text { Cráneo, regente y } \\
\text { guardián de Chel Te' } \\
\text { Chan K'inich (Itzamna- } \\
\text { aj B'ahlam III), el hijo } \\
\text { de P-J IV }\end{array}$ \\
\hline Dintel 50 & & & $\begin{array}{l}\text { Estandarte jasaw chan } \\
\text { sostenido por K'inich } \\
\text { Tatb'u Cráneo Il }\end{array}$ \\
\hline
\end{tabular}

"Seguimos la propuesta de Tate (1997: 96, 244) y Schele y Freidel (1990: 293), pero Mathews (1997: 163) propone la fecha de 26.06.741. 


\section{Rituales de intercambio de estandarte y las orientaciones solsticiales en Yaxchilán}

La importancia de la fecha del solsticio de verano en Yaxchilán se basa en dos tipos de evidencia. Primero, existen las fechas de los rituales del intercambio de estandartes, relacionados con la transferencia del poder; segundo, se encuentran los datos sobre las orientaciones de las estructuras comisionadas por Escudo Jaguar II y Pájaro Jaguar IV. Ambos argumentos fueron retomados por Justeson (op. cit.: 114-115), quien comparó las fechas dadas por Tate con los momentos exactos de dichos solsticios (en calendario gregoriano), y concluyó que más bien se trataba de las fechas que caían inmediatamente después de los solsticios. Puesto que cerca de las fechas solsticiales los puntos de la salida y puesta del sol virtualmente se detienen, los desplazamientos del disco solar resultan tan pequeños que no son perceptibles para el ojo humano. Se puede decir que la mayoría de las fechas apuntaba el día cuando podía observarse el movimiento del sol después del período de la aparente parada. Ello concuerda con la hipótesis de Loundsbury (1982), quien observó una peculiar lógica de los mayas de asociar los eventos importantes con los momentos de partida de los puntos estacionarios (los puntos solsticiales son los puntos estacionarios par excellence). Dicho sea de paso, a este momento podría referirse la danza jasaw chan (Tate, 1986, 1989, 1997).

No obstante, en sus publicaciones Tate (1997: 113) indica que durante el solsticio de verano el sol sale en el punto cuyo acimut alcanza 63 grados. Obviamente las orientaciones de $51^{\circ}$ - $54^{\circ}$ quedan fuera del arco solar de Yaxchilán. El día del solsticio de verano el sol sale y se pone por los puntos más al Norte posible, y es a partir de este momento cuando los puntos de salida y puesta empiezan a moverse hacia el Sur. En otras palabras, los puntos de salida y puesta del sol durante el solsticio de verano nunca se alinearán con los ejes de las estructuras mencionadas. Las orientaciones de dichas estructuras quedan fuera del arco solar y no apuntan a los puntos de salida y puesta del sol en ningún momento del año. En su afán de demostrar el origen solar de la arquitectura de Yaxchilán, Tate resuelve esta dificultad al enfatizar el efecto de la penetración del rayo solar en el interior de la Estructura 33, que ilumina en este momento la estatua de Pájaro-Jaguar IV. Según esta investigadora, después de la salida del sol en 1985 sus rayos iluminaron la estatua del soberano maya durante unos siete minutos (Tate, 1997: 113). De este modo, las orientaciones arquitectónicas, por un lado, y las fechas gregorianas de los rituales del intercambio de estandartes, por el otro, concuerdan entre sí ofreciendo una elegante solución.

Los investigadores que parecen aceptar la idea según la cual Pájaro Jaguar IV utilizó la metáfora solsticial para legitimar su posición como heredero legítimo descendiente de Escudo Jaguar II (por ejemplo, Justeson, op. cit.: 114-115; Schele y Freidle, 1990: 262, 477; Sotelo Santos, 1992: 137; Bardsley, 1994: 90), 
pueden encontrar los argumentos con el mismo tipo de discurso en Palenque, en donde la designación de Kan B'ahlam II como heredero de Janaab' Pakal I también ocurrió durante el solsticio de verano, el 22.06.741 (Bardsley, op. cit.). ${ }^{2}$

\section{Estructura 33}

La Estructura 33 se sitúa en el tercer nivel de los edificios a los cuales se llega ascendiendo desde la parte central de la Gran Plaza hasta la Gran Acrópolis (Estructuras 37 y 38, Sotelo, op. cit.: 95-98). Su planta rectangular mide 22 por 4.88 $\mathrm{m}$ y su altura alcanza $13 \mathrm{~m}$ (incluida la crestería, Sotelo, op. cit.: 104). ${ }^{3}$ El edificio tiene tres entradas, con tres dinteles. En su interior se encuentra una estatua sedente que representa a Pájaro Jaguar IV (Mathews, op. cit.: 204-205).

La Estructura 33, atribuida a Pájaro Jaguar IV, posiblemente fue terminada por su hijo, Itzamnaaj B'ahlam III (Martin y Grube, op. cit.: 132-133), quien reinó a partir de 769 y alrededor de 800 . Cada uno de los tres dinteles representa a Pájaro Jaguar participando en una danza ritual. El Dintel 2 muestra a Chel Te' Chan K'inich, el futuro Itzamnaaj B'ahlam III, en un ritual de baile con su padre, Pájaro Jaguar IV, sosteniendo en las manos un cetro conocido como xukpi, que representa al árbol con pájaro (Freidel et al., op. cit.: 271). No obstante, su fecha 9.16.6.0.0 (Mathews, op. cit.: 203) corresponde a la fecha gregoriana del 7 de abril de 757, y nada tiene que ver con el solsticio de verano. Cabe decir que es ésta la última fecha asociada con la Estructura 33 (ibid.).

\section{Orientaciones de la Estructura 33}

Durante la temporada de campo en 2001 realizamos una serie de mediciones astronómicas en torno de la Estructura 33. Hemos establecido que sus paredes

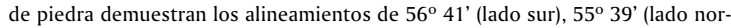
te) y $144^{\circ} 41^{\prime}$ (lado este). Ya que se trata del fenómeno de la entrada de los rayos solares hacia el interior de la estructura, en adelante nos interesará la fachada oriental del edificio. La línea perpendicular a la fachada trazada hacia el este tiene el acimut de $54^{\circ} 41^{\prime}$, lo que está de acuerdo con los datos de Tate.

\footnotetext{
${ }^{2}$ Esta investigadora insiste en relacionar la fecha del intercambio de estandartes en la estela 11 con la conjunción inferior y la salida heliaca de Venus. No obstante, la conjunción inferior de Venus ocurrió en 11.06.741 (fecha gregoriana, Meeus, 1995: 415), 15 días antes de la fecha indicada, lo que descarta cualquier intento de relacionar la fecha con los movimientos venusinos.

${ }^{3}$ En su libro recién publicado García Moll (2003: 205) ofrece las medidas definitivas del Edificio: $22.2 \mathrm{~m}$ de largo por $4.8 \mathrm{~m}$ de ancho. Es de notar que según este investigador su orientación es de $45^{\circ}$ (García Moll, op. cit.: 307) lo que constituye un evidente error tipográfico (en lugar de $45^{\circ}$ debe ser $54^{\circ}$ ).
} 
No obstante, la altura del horizonte observada tiene $1^{\circ} 10^{\prime}$, lo cual implica que la salida del sol en el momento del solsticio de verano se percibe en la dirección de $65^{\circ} 41^{\prime}$, es decir, $2^{\circ} 41^{\prime}$ al sur del punto señalado por dicha investigadora (1997: 113). La posición del dintel limita la altura del sol observada desde el lugar donde se sitúa la estatua de Pájaro Jaguar IV, de tal manera que los rayos solares pueden penetrar al interior sólo cuando el sol se halla a una altura menor a $9^{\circ}$. Esta es la altura del dintel vista desde la posición de dicha estatua. Hemos calculado que cuando el sol se encuentra a la altura de $9^{\circ}$ sobre el horizonte, su acimut es de $67^{\circ} 53^{\prime}$. Por lo tanto, las ilustraciones que Tate publica en sus trabajos (1989: 419, figuras 32.2a y 32.2b; 1997: 113, figuras 43a y 43b) más bien reflejan sus ideas y no una situación real.

Ahora bien, como ya se ha dicho, la orientación de la fachada de la Estructura 33 impide observar directamente la salida del sol durante el solsticio de verano. El punto de la salida del sol está desviado por casi $11^{\circ}$ de la línea trazada perpendicularmente con respecto al eje de la fachada. De manera que, los rayos solares, que en efecto entran en la estructura, iluminan sólo una parte de la estatua de Pájaro Jaguar IV. Debido a que su trayectoria es sesgada y no permite iluminar directamente la fachada de la estatua, hay que poner en duda la intencionalidad de los arquitectos mayas en resaltar el momento solsticial. A. P. Maudslay (1889-1902, vol. II.: 46), quien visitó (y descubrió) Yaxchilán en 1883, describe el hallazgo de la estatua de bulto de Pájaro Jaguar IV dentro de la Estructura 33. Este estudioso reporta que dicha estatua fue hallada en el nicho opuesto a la puerta central de la Escultura 33 y cerca de ella se encontraba su cabeza rota. Resulta que la estatua fue colocada en el interior del nicho, separado por la crujía transversal de la puerta de acceso. Con una orientación sesgada de la fachada con respecto a la posición del sol durante los primeros minutos después de su salida en el día del solsticio de verano, es imposible que los rayos penetren en el nicho a lo largo y ancho, tal como lo sostiene Tate. Si la intención de los constructores de la Estructura 33 fue la de colocar la estatua dentro del templo con el fin de iluminarla con los rayos del sol solsticial, parecería más lógico orientarla hacia el punto en donde se percibe la salida del sol.

\section{Conclusión}

Estas circunstancias, junto con el hecho de que las Estructuras 20, 21, 40 y 41 exhiben alineamientos que se sitúan lejos de la posición solsticial del sol (véase tabla 2), nos obligan a cuestionar el planteamiento original de Carolyn Tate. Todas estas estructuras no se alinean con la salida del sol en el solsticio de verano. Por lo tanto, se derrumba uno de los argumentos más importantes en favor del origen solar del arreglo arquitectónico del centro ceremonial de Yaxchilán. 
Tabla 2. Datos sobre las orientaciones de las estructuras 20, 21, 40 y 41

\begin{tabular}{|c|l|c|c|c|c|}
\hline \multicolumn{1}{|c|}{ edificio } & \multicolumn{1}{|c|}{$\begin{array}{c}\text { Tate } \\
(1997: 187, \\
197,240)\end{array}$} & $\begin{array}{c}\text { Tate } \\
(1997: \text { 112) }\end{array}$ & $\begin{array}{c}\text { García } \\
(2003: 306, \\
308)\end{array}$ & $\begin{array}{c}\text { acimut 1 } \\
\text { (pared } \\
\text { corta) }\end{array}$ & $\begin{array}{l}\text { acimut 2 } \\
\text { (fachada) }\end{array}$ \\
\hline Estructura 20 & $49^{\circ}-53^{\circ}$ (brújula) & $53^{\circ}$ & $40^{\circ}$ & $58^{\circ} 56^{\prime}$ & $323^{\circ} 44^{\prime}$ \\
\hline Estructura 21 & $50^{\circ}$ (brújula) & $54^{\circ}$ & $42^{\circ}$ & $50^{\circ} 03^{\prime}$ & $318^{\circ} 41^{\prime}$ \\
\hline Estructura 40 & no hay & $53^{\circ}$ & $50^{\circ}$ & $55^{\circ} 04^{\prime}$ & $327^{\circ} 42^{\prime}$ \\
\hline Estructura 41 & $\begin{array}{l}49^{\circ}-51^{\circ} \text { (brújula) } \\
54^{\circ}-57^{\circ} \text { (mapa) } \\
322^{\circ} \text { fachada }\end{array}$ & $51^{\circ}$ & $50^{\circ}$ & $54^{\circ} 22^{\prime}$ & $324^{\circ} 22^{\prime}$ \\
\hline
\end{tabular}

Los datos publicados por Tate (1997) y García Moll (2003) se comparan con las medidas hechas en campo.

\section{BIBLIOGRAFÍA}

Bardsley, Sandra Noble

1994 "Rewriting history at Yaxchilan: inaugural art of Bird Jaguar IV", en Seventh Palenque Round Table, 1989, pp. 87-94, Virginia M. Fields (ed.). San Francisco: The Pre-Columbian Art Research Institute.

Freidel, David, Linda Schele y Joy Parker

1993 Maya Cosmos. Three Thousand Years on the Shamans Path. Nueva York: William Morrow.

García Moll, Roberto

2003 La arquitectura de Yaxchilán. México: INAH y Plaza y Valdés.

Grube, Nikolai

1992 "Classic Maya dance: evidence from hieroglyphs and iconography", Ancient Mesoamerica (3): 201-218. Nueva York: Cambridge University Press.

Justeson, John S.

1989 "Ancient Maya ethnoastronomy: an overview of hieroglyphic sources", en World Archaeoastronomy, pp. 76-129, A. F. Aveni (ed.). Cambridge: Cambridge University Press.

Looper, Matthew G.

2003 "The meaning of the Maya flapstaff dance", Glyph Dwellers (17): 1-4.

Loundsbury, Floyd G.

1982 "Astronomical knowledge and its uses at Bonampak, Mexico", en Archaeoastronomy in the New World, 143-168, A. F. Aveni (ed.). Cambridge: Cambridge University Press. 
Martin, Simon

2004 "A broken sky: the ancient name of Yaxchilan as Pa' Chan", The PARI Journal 5 (1): 1-7. Lafayette, California: Precolumbian Art Research Institute.

Martin, Simon y Nikolai Grube

2000 Chronicle of the Maya Kings and Queens: Deciphering the Dynasties of the Ancient Maya. Londres: Thames and Hudson.

Mathews, Peter Lawrence

1997 La escritura de Yaxchilán. México: INAH (Colección Científica, 368).

Maudslay, A. P.

1889-1902 Biología Central-Americana, editado por F. Ducane Godman y Osbert Salvin.

Archaeology (edición facsimilar de F. Robicsek, Milpatron, Nueva York, 1974). Londres: Porter, 5 vols.

Meeus, Jean

1995 Astronomical Tables of the Sun, Moon and Planets, 2a. ed., Richmond, Virginia: Willmann-Bell.

Miller, Mary

2001 "Life at court: the view from Bonampak", en Royal Courts of the Ancient Maya (2): 201-222, Takeshi Inomata y Stephen D. Houston (eds.). Boulder: Westview.

Proskouriakoff, Tatiana

1963 "Historical data in the inscriptions of Yaxchilan, part 1. The reign of ShieldJaguar”, Estudios de Cultura Maya, 3: 149-167. México: UNAM, IIFL, Centro de Estudios Mayas.

Schele, Linda y David Freidel

1990 A Forest of Kings. The Untold Story of The Ancient Maya. Nueva York: William Morrow.

Sotelo Santos, Laura Elena

1992 Yaxchilán. México: Gobierno del Estado de Chiapas.

Tate, Carolyn

1986 "Summer solstice ceremonies performed by Bird-Jaguar III of Yaxchilan, Chiapas", Estudios de Cultura Maya, 16: 85-112. México: UNAM, IIFL, Centro de Estudios Mayas.

1989 "The use of astronomy in political statements at Yaxchilan", en World Archaeoastronomy, pp. 416-429, A. F. Aveni (ed.). Cambridge: Cambridge University Press.

1997 Yaxchilan. The Design of a Maya Ceremonial City. Austin: University of Texas Press. 


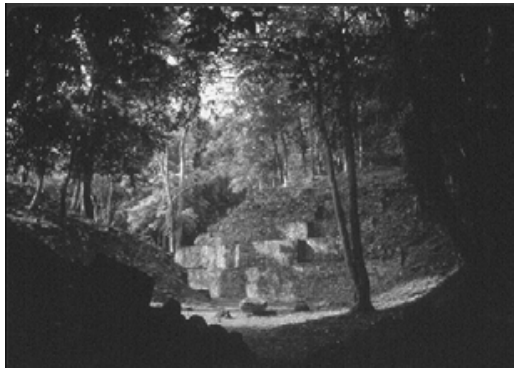

Figura 1. Vista de Yaxchilán en su acceso noroeste

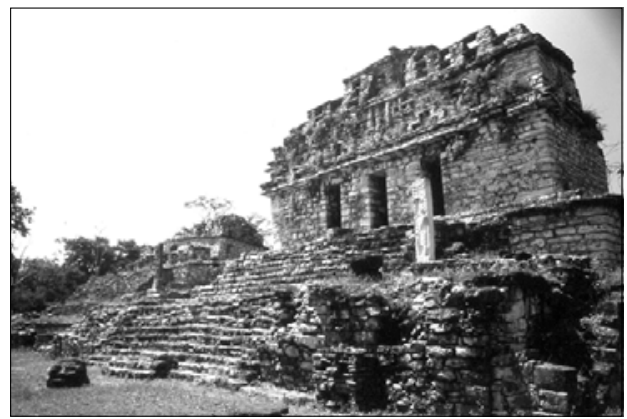

Figura 2. Estructuras 40 y 41 , en la cúspide de la ciudad 


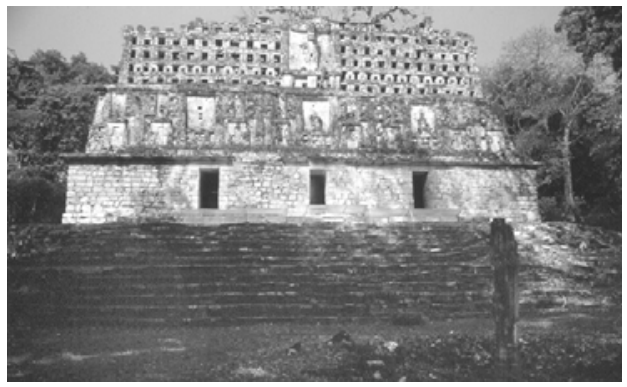

Figura 3. Estructura 33 de Yaxchilán, con una estela labrada en una estalactita

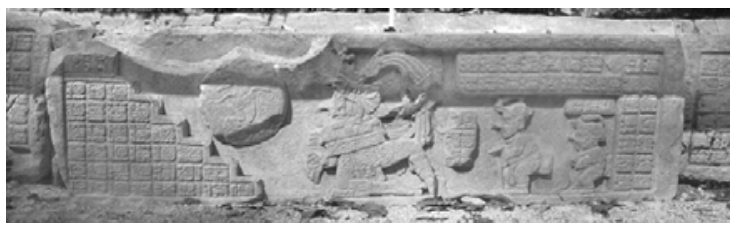

Figura 4. Relieve con escena del juego de pelota, en el acceso a la Estructura 33 de Yaxchilán 


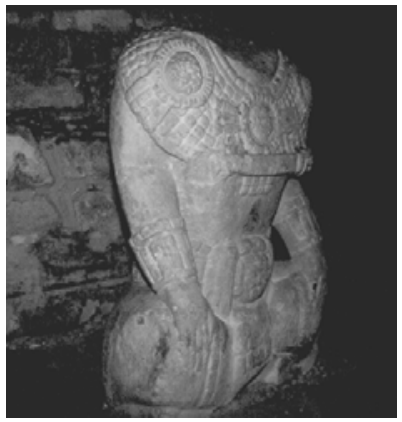

Figura 5. Escultura del soberano Pájaro Jaguar IV, en el interior de la Estructura 33

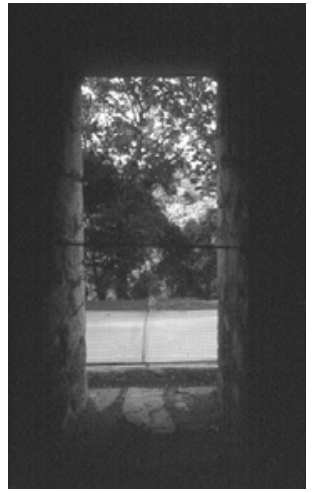

Figura 6. Vista hacia el oriente desde la escultura de Pájaro Jaguar IV, a través del vano central de la Estructura 33 


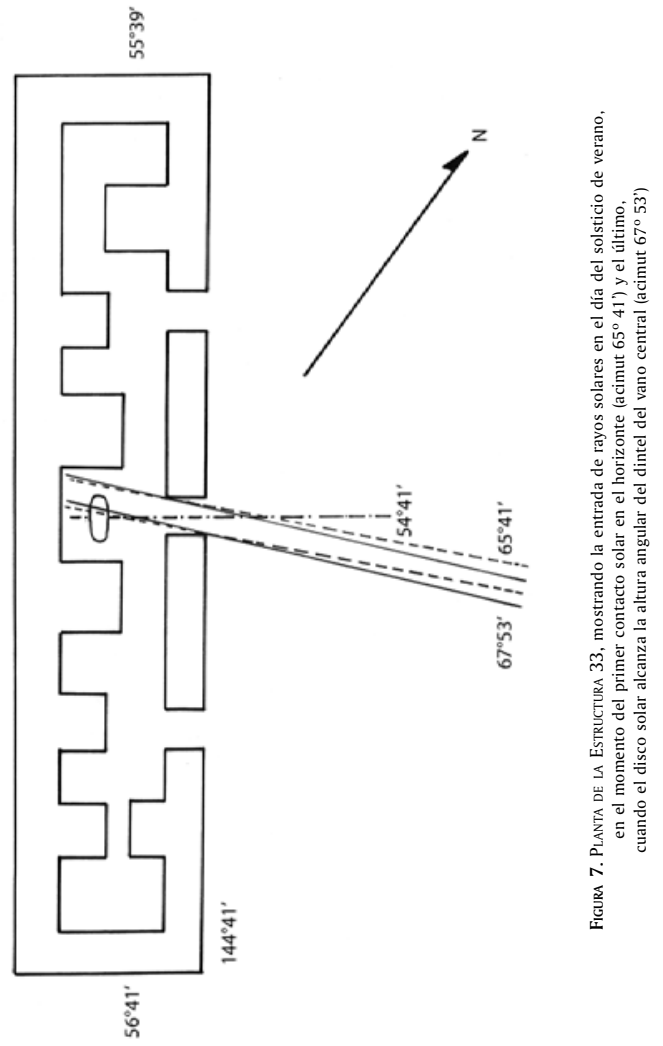

